\title{
Evaluation of low cost capacitive ECG prototypes: a hardware/software approach
}

\author{
Jörg Güttler; Christos Georgoulas; Thomas Linner; Thomas Bock \\ Chair for Building Realization and Robotics \\ Technical University Munich, Germany \\ joerg.guettler@br2.ar.tum.de
}

\begin{abstract}
Demographic change is greatly affecting more and more industrial nations. Therefore, healthcare solutions become increasingly interesting for end-users. However, existing solutions like fitness trackers are mostly worn for a short period of time, i.e. during exercising. Therefore, the implementation of unobtrusive biological sensors via capacitive Electrocardiography (ECG), for application within the user home environment, becomes interesting in the Ambient Assisted Living (AAL) research areas. A capacitive ECG has been developed using an Arduino/Wattuino Uno microcontroller in order to enable the realization of a low cost capacitive ECG System, using existing of-the-shelf components. The developed system comprises an improved version of an earlier prototype of the authors, differing in both hardware and software components used. Furthermore, wireless data transmission quality has been here investigated, in order to enable an unobtrusive implementation of the proposed system when it is used in home environments. In this paper the authors investigate and compare the quality of the measured ECG signal quality under different hardware and software configurations. The results in the paper show that using XBee modules as a secure wireless data transfer solution has a significant deterioration of the measured signal quality. Therefore, solutions are proposed and discussed in the paper to overcome the aforementioned limitations.
\end{abstract}

Keywords- ECG; Capacitive Electrodes; XBee; microcontroller; FIR filtering

\section{INTRODUCTION}

In industrial nations angiopathy, which leads to coronary heart diseases, heart attacks, or strokes, comprises one of the main death reason. The metabolic syndrome has shown a strong relation to these diseases [1]. These have of course a genetic impact (e.g. sex, genetic preposition, high age, etc.), however there are also influenceable risk factors. According to $[2,3]$ a poor lifestyle behavior is mostly the reason for these diseases. Behavior factors like smoking, stress, physical inactivity, nutrition, and obesity, are the factors, which can be actively influenced by the user [1]. By detection of early symptoms, which are at the beginning silent, the user can be enabled to start a prevention (also on the physician level), before more evident symptoms occur, because then it is mostly too late to maintain the life quality of the affected. Furthermore, demographic change is currently affecting many industrial nations. The elderly population of elderly is rapidly increasing while birth rates are dropping. This effect results in an increasing numbers of health issues among the population [4]. Care home facilities and hospitals are facing challenging problems, therefore, a major research topic is the development of systems, which could prevent the user from age related diseases, e.g. coronary heart disease, strokes etc.

The increased interest in lifestyle and health style products lead to a large variety of apps and programs, which support the user in a healthy life style. Also several wearable sensors are playing an increased role, supported by lifestyle apps and smartphones [5]. However, wearables tend to be forgotten by the user after some time and finally, the bad habits of the user are coming back and the prevention of the aforementioned diseases fails. Therefore, the authors are focusing on a different approach. Instead of further developing wearables, the measurement of biological signals should be implemented in the environment of the user. The measurement should be as unobtrusive as possible. Additionally, the price of lifestyle, health, and AAL systems matters, as the users have to be able to afford the proposed systems. Larger systems, e.g. as proposed by $[6,7]$, use the modularity to extend the services of the overall system according to the user needs and financial situation. For this purpose, the authors already developed in an earlier work [8] a prototype of a low cost capacitive ECG, including a wireless data transfer option. Due to its compact size it can be unobtrusively embedded in a bed or chair. Acquired measurements can be wirelessly transferred and stored to a gateway system for further use, e.g. to trigger an emergency call, to be evaluated by a physician, or to automatically estimate and predict the current health condition. However, tests with the first prototype [8] revealed several challenges, i.e. ambient noise affecting the measured signal, capacitive charging, low sampling frequency etc. These findings lead to the modification of the proposed system architecture and to the realization of a second prototype which is proposed in this paper. The authors present here an overview of the improved capacitive ECG system and its components, and compare the different hardware and software solutions, to optimize the proposed low cost capacitive ECG system.

\section{PROPOSED CAPACITIVE ECG PROTOTYPING}

Both the old and refined prototypes are built using a similar approach. The capacitive ECG System consist out of three main parts: a) Capacitive electrodes, b) processing unit, 
and c) user interface (shown in Fig. 1). The capacitive electrodes, also called active electrodes $[9,10,11]$, can be seen as a capacitive surface, which leads to the coupling capacitance with the human body. The impedance of the active electrodes should range between $10 \mathrm{~T} \Omega$ and $100 \mathrm{~T} \Omega$ [12]. To achieve this large amount of impedance an operational amplifier like the OPA602AP [13] can be used.

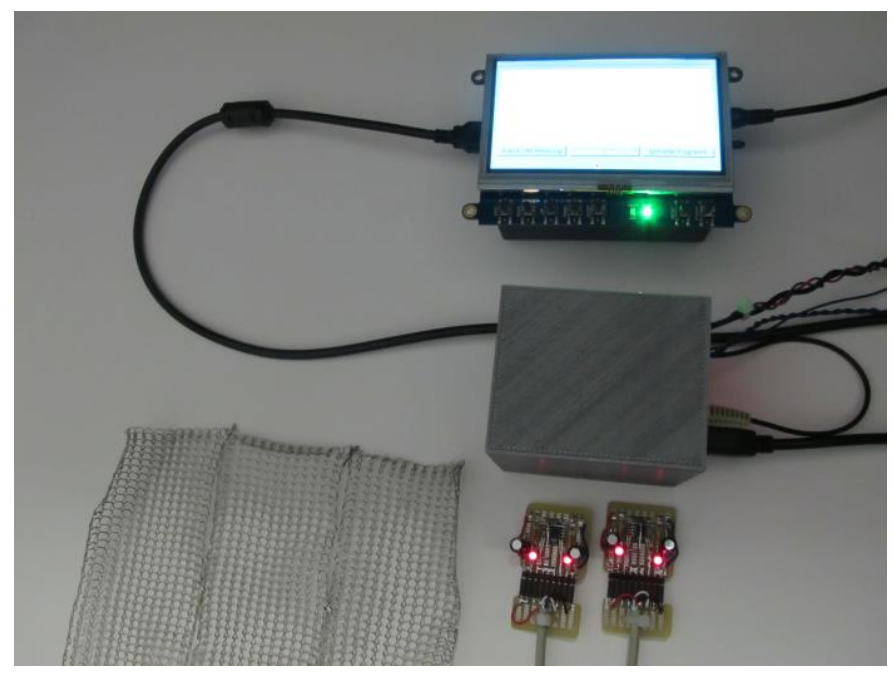

Fig. 1. Proposed capacitive ECG; top is the user interface which is by USB connected to the processing unit in the middle; at the bottom the three electrodes can be seen.

Capacitive ECG electrodes can be already found as purchasable products out on the market, i.e. like the ones embedded in driver seats [14]. Active electrodes can also be already bought in an integrated circuit arrangement package (chip). The authors utilized two different types of active capacitive electrodes: the PS25203B [15] and the PS25201B [16]. The interface between the active electrodes and the different processing units (Fig. 2), has been developed in a way to allow an easy swap of the two different electrodes among the two processing units, for straightforward testing purposes.

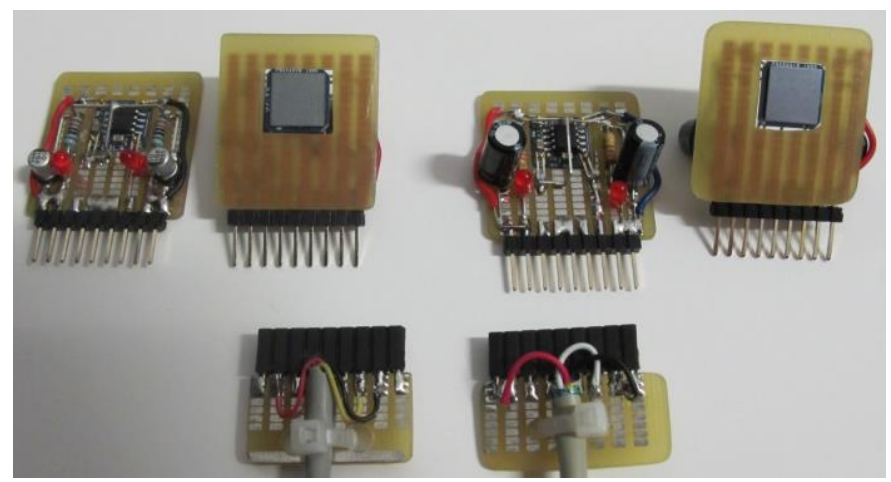

Fig. 2. Top and botom views of the proposed active electrodes; left PS25203B and on the right the PS25201B; connector intefaces appear on the bottom

The processing unit consists out of the Arduino/Wattuino Uno, an XBee shield, and an ECG shield. To solve the issue of the missing ground of the capacitive ECG the "Driven-
Ground-Plane" circuit, as proposed from [17], has been built as a shield, which was directly attached on the processing unit. This is a common way to filter out unwanted noise, and is therefore a corresponding replacement for the ground electrode. The XBee shield comprises the interface for the XBee Pro Series 2B module to the Arduino Board [18]. Thereby, the measurements can be directly wirelessly transferred, e.g. to a terminal, or user interface. Both proposed prototypes (visible in Fig. 3) differ only in the used shield for capturing the signal, besides also some implementation improvements of the "Driven-Ground-Plane" circuit. The first prototype [8] is using the e-Health shield from Cooking Hacks [19], whereas the second prototype is using the "SHIELDEKG-EMG" shield from Olimex [20]. These shields interface the Arduino with the electrodes (passive as well as active electrodes) and consist out of several analog filters, and the necessary differential amplifier. Furthermore, the e-Health shield from Cooking Hacks includes further interfaces for different devices (like blood sugar, breath etc.), whereas the ECG shield from Olimex focuses on the ECG and supports also a 6 lead ECG application (whereas for each channel is an own shield necessary).

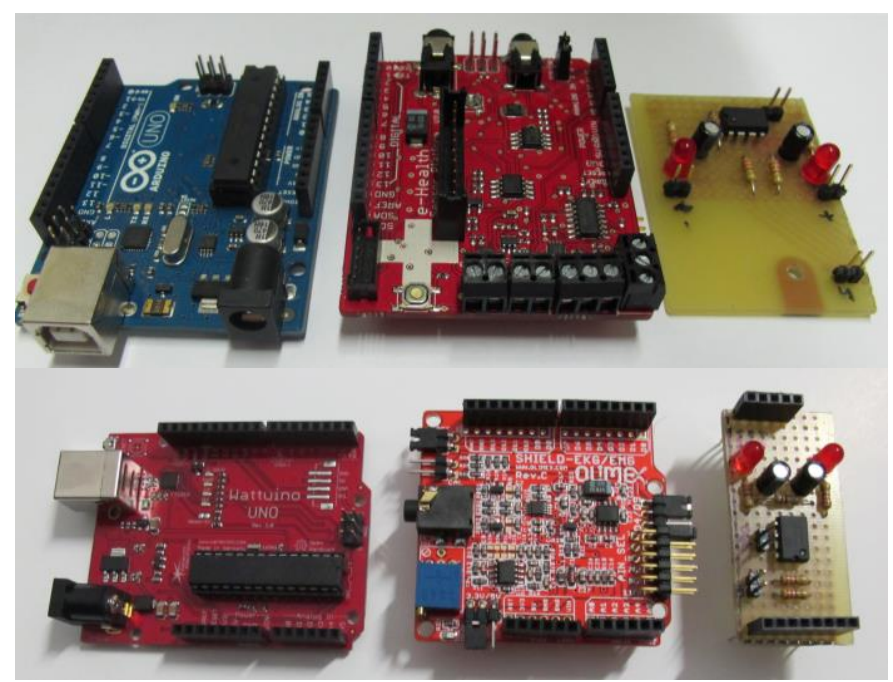

Fig. 3. AT the top are the parts of the first [8] prototype of the processing unit visible, consisting out of Arduino Uno, e-Health shield and "DrivenGround-Plane" circuit shield; at the bottom the hardware parts of the second prototype consisting out of Wattuino Uno, ECG shield from Olimex and the "Driven-Ground-Plane" circuit shield (redesigned).

As user interface the single board computer BeagleBone Black [21] has been used. The hardware settings for the user interface are for both prototypes unaltered and consist out of a shield, which interfaces the XBee module with the BeagleBone Black, and a touch LCD screen monitor. However, on the BeagleBone Black the software for visualizing the measurement needs to adapt, according to revised the hardware settings.

\section{Proposed USER INTERFACE APPLICATION}

The algorithm for the user interface application is depicted in Fig. 4. For plotting and data visualization on the onboard LCD screen the "QCustomPlot" C++ widget has been used [22]. The development has been performed on an Ubuntu 
14.04 platform using QT version 4.8. The surface of the program has been kept as simple as possible, utilizing three buttons (start, stop measurement and close application), in order to be intuitive enough for elderly people. As the BeagleBone Black has only one USB interface available, the necessary communication interface has been hardcoded, using QExtSerialPort. Also the XBee shield is only supporting one XBee module, whose port (/dev/ttyO2) must be activated [23]. This anyhow has been automated by corresponding scripts on the BeagleBone Black.

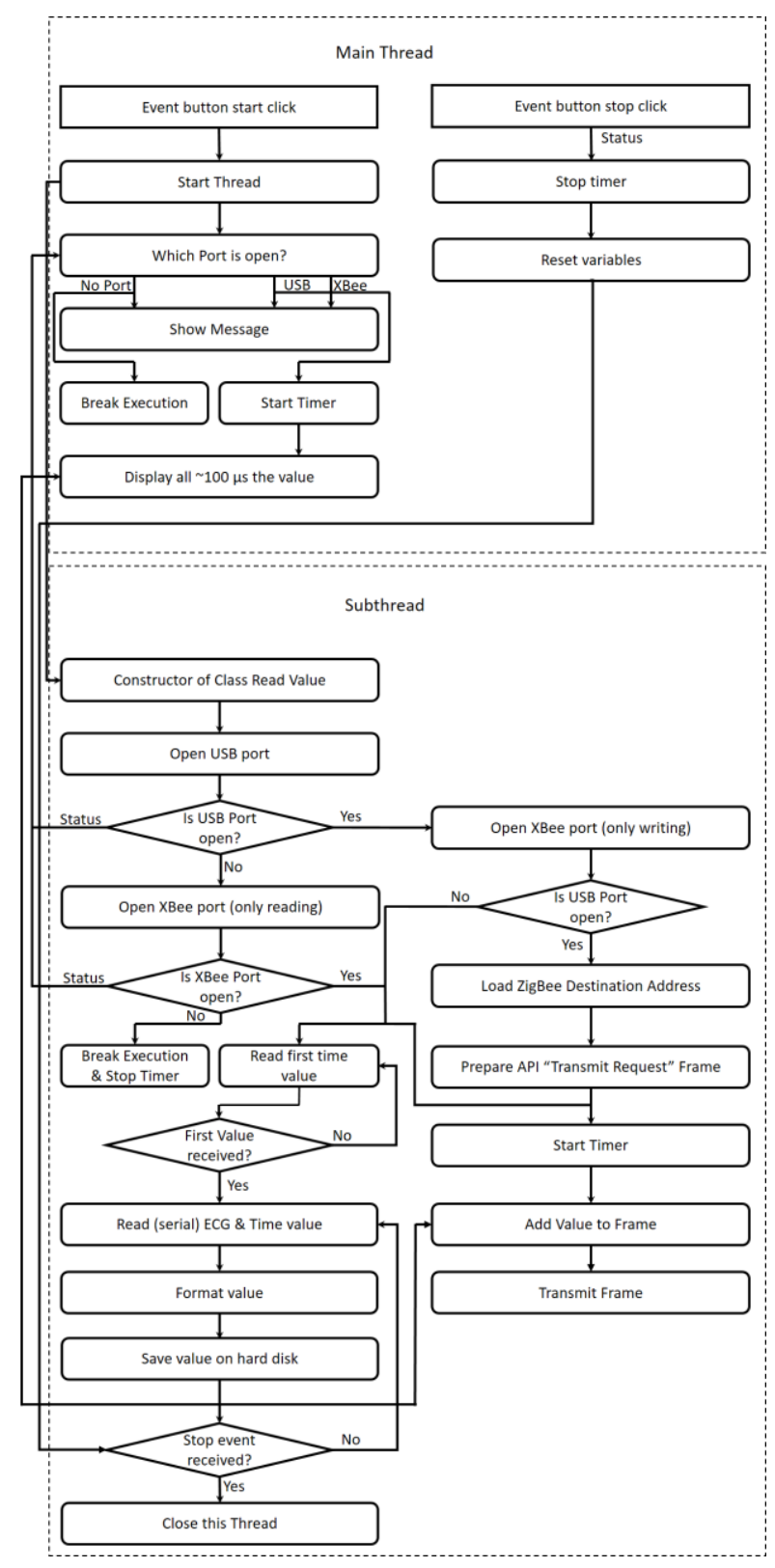

Fig. 4. Proposed user interface algorithm

As soon as the user initiates a measurement, the program is initially checking whether there is a physical connection over USB between the processing unit and the user interface BeagleBone Black board. If not, the program will automatically check, if an XBee module (wireless connection) is already established in order to switch to it and display acquired data. In case the USB connection is present and active, the program is also checking in parallel if an XBee module is attached (or activated) and it uses this additional communication link to forward the measurements to another XBee module. XBee modules provide two different data transmission modes, the transparent (AT) mode, and the Application Programming Interface (API) mode [23]. The transparent mode has to be handled like a serial interface. However, the proposed system is only using the API mode. Tests conducted with the first prototype [8] showed an improved reliability on the data transmission. This is due to the API frame structure depicted in Fig. 5.

\begin{tabular}{|c|c|c|c|c|}
\hline 1Byte & 0x7E & & \multicolumn{2}{|l|}{ Start Delimiter } \\
\hline 2Byte & $0 \times 10$ & $0 \times 01$ & \multicolumn{2}{|l|}{ Length of the frame } \\
\hline 1Byte & $0 \times 10$ & & \multicolumn{2}{|l|}{ Frame type } \\
\hline 1Byte & $0 \times 10$ & & \multicolumn{2}{|l|}{ Frame ID } \\
\hline 4Byte & $0 \times 00$ & $0 \times 13$ & $0 \times A 2 \quad 0 \times 00$ & Destination Serial Number High \\
\hline 4Byte & $0 \times 40$ & $0 \times C 4$ & OxCB $0 x B 9$ & Destination Serial Number Low \\
\hline 2Byte & OxFF & OXFE & \multicolumn{2}{|c|}{ 16-Bit address (if unknown) } \\
\hline 1Byte & $0 \times 00$ & & \multicolumn{2}{|l|}{ Broadcast radius } \\
\hline 1Byte & $0 \times 00$ & & \multicolumn{2}{|l|}{ Options } \\
\hline 19Byte & \multicolumn{4}{|c|}{ Storage for the message } \\
\hline 1Byte & & & \multicolumn{2}{|c|}{ Checksum, which must be calculated } \\
\hline
\end{tabular}

Fig. 5. API frame structure used for transmitting information over XBee

The proposed modules are only sending the frame, which contains the data, if the checksum is correct. The checksum can be calculated according to equation 1 [23].

$$
\text { Checksum }=0 \mathrm{xFF}-\left[\left(\sum_{i=3}^{n-1} \operatorname{Frame}(i)\right) \& 0 \mathrm{xFF}\right]
$$

Furthermore, the message frame allows addressing a specific target XBee module. Although the XBee modules are able to broadcast, the transmission speed is much too slow for streaming the ECG by XBee.

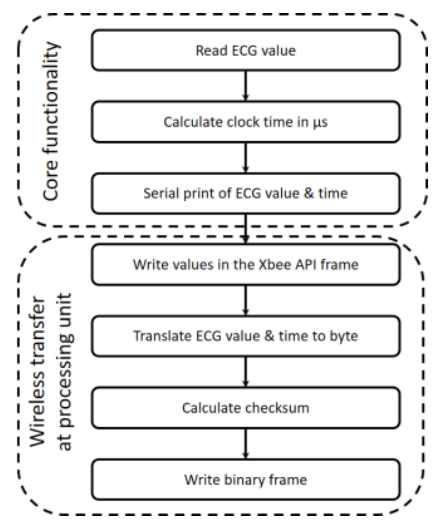

Fig. 6. The Arduino/Wattuino Uno proposed algorithm, consisting out of the core funtionallity and the additional wireless data transmission routines using the XBee API mode. 
Only with unicast transmission the sample frequency is appropriate to stream the ECG signal. In order to make the program able to adapt, according to the environmental implementation, a configuration file containing the destination addresses, which is loaded on application initialization is used. Thereby maintenance personnel can modify the transmission destinations in case modifications are required. The Arduino code of the processing unit is capturing the data, and forwarding either to the USB port, and - if an XBee module is directly attached to the Arduino/Wattuino Uno - additionally to the XBee port, which address is directly hardcoded in the Arduino code. Especially for the second case, the algorithm is depicted in Fig. 6.

\section{RESULTS AND DISCUSSION}

Experiments conducted with the proposed capacitive ECG systems have proven that the XBee modules, the ECG shields and the electrodes impose an adequate influence on the acquired signal quality. The shield and the electrodes influence manly the amplitude of the signal, however the XBee modules introduce a negative effect on the sampling frequency, and thereby the acquired signal of the proposed system.

\section{A. XBee modules related deteriorated performace findings}

Several tests of the proposed system showed that the XBee modules deteriorate the quality of the acquired signal. This can be observed by comparing the measurement quality of the processing unit without and with the XBee module (Fig. 7).

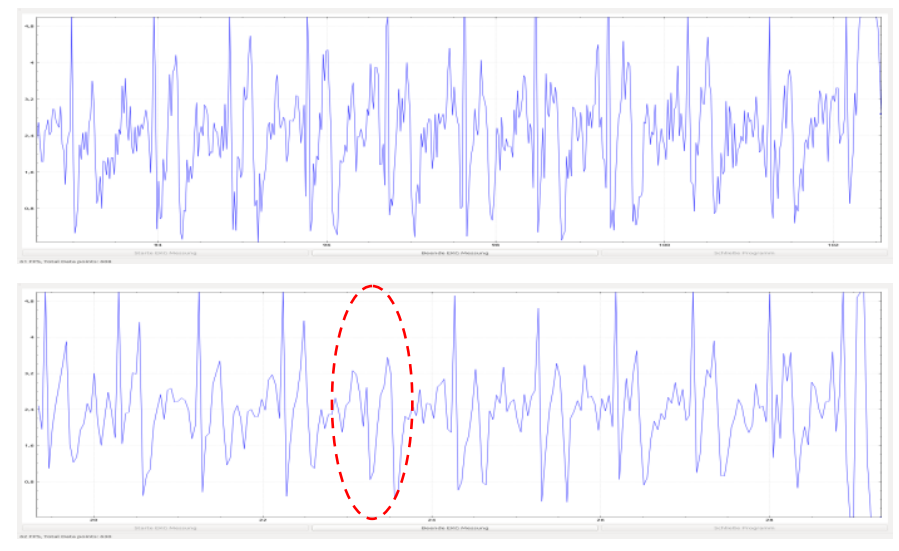

Fig. 7. ECG measurment Eindhoven I, measurement points hands, direct skin contact; at the top measuremnt without an XBee antenna and a sample frequency of $53 \mathrm{~Hz}$; bottom XBee transmission from Arduino with $24 . \mathrm{Hz}$ sample frequency.

As it is visible in Fig.4, the plotting of the measured signal routine runs independently from the data storing routine. This is necessary, as the Arduino transmission (over USB) is providing additional redundant data than the BeagleBone Black can plot. The timer can be seen as a down sampling of the visual feedback. However, comparing the stored measurements, it was confirmed that the processing unit sample frequency is decreasing, if the XBee module is active. Without an XBee module within 110 Seconds, around 6000 samples can be registered, which corresponds to a sample Frequency of about $53 \mathrm{~Hz}$. If the XBee module is sending the data stream at the same time, the sample frequency of the stored data is dropping to about $24 \mathrm{~Hz}$, and thereby leads to a reduced acquired signal quality. The human heartbeat normally ranges between 60 to 80 beats per minute (in stress it can easily increase up to 120 beats per minute). Heart attacks with ventricular heart flickering can cause more than 350 beats per minute [24]. This physiological values show that an ECG system should at least support a sample frequency of about $25 \mathrm{~Hz}$ in order not to miss a beat. However, the ECG signal is a complex biological signal, and already a sample frequency of $24.20 \mathrm{~Hz}$ is influencing the ECG signal shape. In Fig. 9 (bottom) it can be seen that already the $\mathrm{R}$ wave (which marks the ventricular main contraction) is at least once missing, due to the low sampling frequency. In order to improve the signal quality, to be at least close to $50 \mathrm{~Hz}$, the Arduino algorithm, shown in Fig. 6, was modified, in order to transmit only each $4^{\text {th }}$ recorded sample. The result was an increased sample frequency on the USB port, with more than $48 \mathrm{~Hz}$. However, on the other side, the XBee module has been down sampled to around $11 \mathrm{~Hz}$. As it can be seen in Fig. 8, the signal quality is heavily deteriorated.

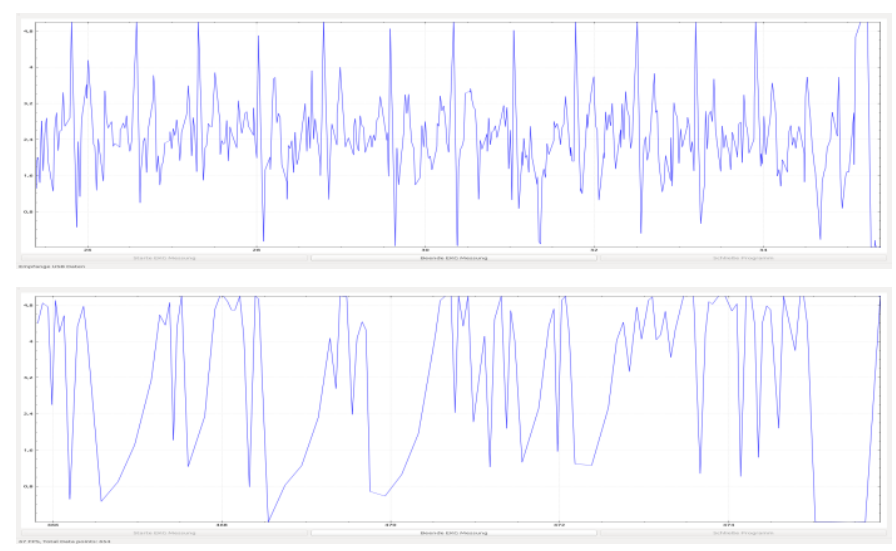

Fig. 8. ECG measurment, top received data from USB port with $48.01 \mathrm{~Hz}$ sample frequency, bottom received data from the Arduino XBee transmission with $11.28 \mathrm{~Hz}$ sample frequency.

As the Arduino/Wattuino Uno is not supporting multithreading processing [25], there were not many possibilities in improving the signal quality on the processing unit. However, the BeagleBone Black supports, also on the hardware level, multi-threading. As seen in Fig. 4, the subthread for data polling of the processing unit, is triggering a second timer, which is only responsible for forwarding the received data (independent from the plotting routine). The sample frequency is still slightly dropping to almost $53 \mathrm{~Hz}$, which is very close to the sample frequency without any XBee transmission. However, compared to the down sampling approach visible in Fig. 8, the sample frequency of the XBee module is here almost $25 \mathrm{~Hz}$. This means, the signal quality by this approach can effectively improve, without reducing the low sample frequency of the XBee modules. Comparing AT and API modes, showed no significant difference regarding the sample frequency. 

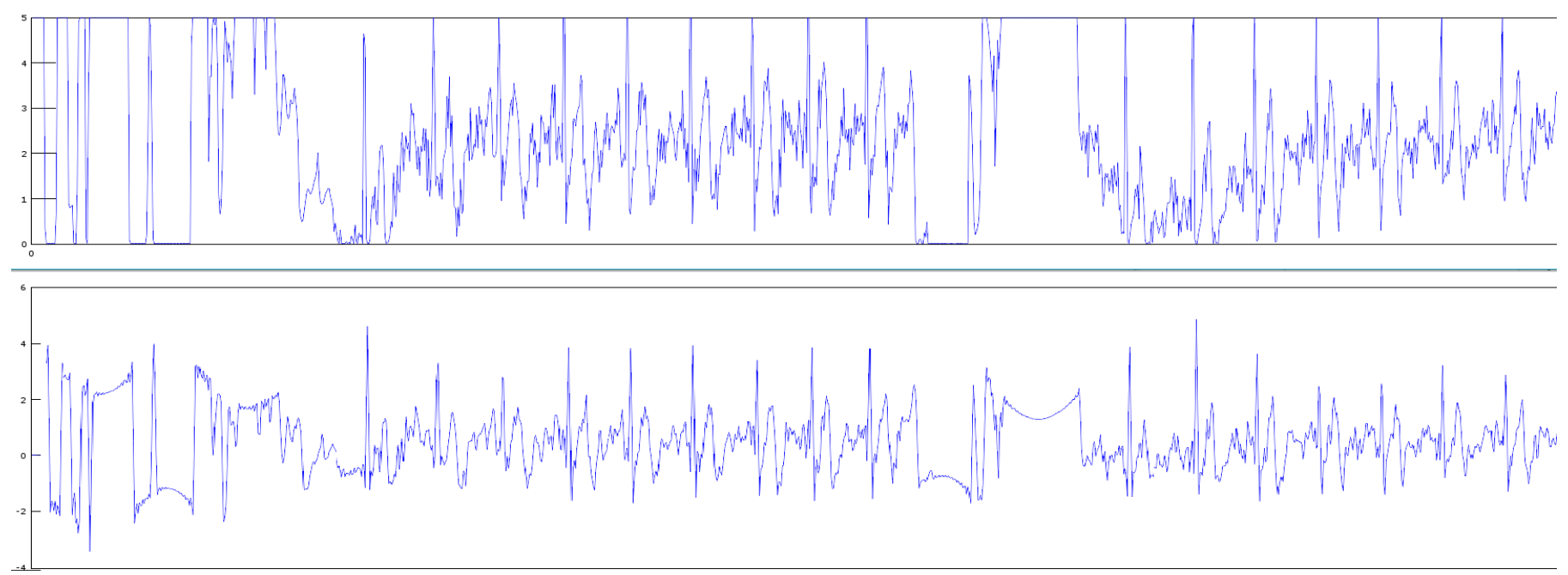

Fig. 9. Captured ECG signal including some artefacts; top is the raw signal as captrued and stored in the user interface; bottom is the FIR filtered signal by using GNU Octave interpreter language (mesurements were made over direct skin contact on the hands)

\section{B. Shields related improved performace findings}

According to [26] the Olimex ECG shield comprised several filters, a differential amplifier as well as an operation amplifier with regulated gain, which leads compared to normal electrodes to an improved amplitude in the signal. Also the eHealth shield from Cooking Hacks comprises some onboard analog filters, as well as a differential amplifier. Therefore, under same conditions, both shields only differ in the amplitude. As the Olimex ECG is amplifying the signal too heavily for the active electrodes, the power supply of the Olimex ECG shield has been set to $3.3 \mathrm{~V}$. Even then the amplitude is higher than the measurement of the e-Health shield. The implemented analog filters improve the signal quality that even an FIR filter of order 128 is minor improving, as seen in Fig. 9. Fig. 9 represents the logged data from the ECG System into GNU Octave, convoluting the signal with an FIR band pass filter $0.75 \mathrm{~Hz}$ to $40 \mathrm{~Hz}$. The results show that there is an improvement of the captured signal. However, the containing information is not changed. The ECG signal contained within the noise or artefacts signal cannot be recovered by the FIR filter. Therefore, in the application no FIR filtering algorithm has been implemented yet, as the contribution of such a filter is minor. However, using a buffer and a fast Fourier algorithm [27] for the convolution part, the signal quality can be optimally improved.

\section{Influence related to the active electrodes}

Both electrodes are similar constructed as in Fig. 2 shown. However, they differ in the signal pre-amplification. This means that PS25203B is not compatible with PS25201B. Each channel must use the same electrode chip in order to receive a capacitive ECG. PS25203B has a lower amplitude because of the reduced amplification, however the discharging of this electrode needs less time, compared to the PS25201B. This is very useful especially in the case where the electrodes are used as dry electrodes. Both electrodes have been able to measure through clothes (e.g. shirt and sweaters). The measurement results are depicted in Fig. 10. The increased amplitude of the measurement with the PS25201B electrode allows also to measure through thick cloths. On the other side, the PS25203B is less affected by noise.

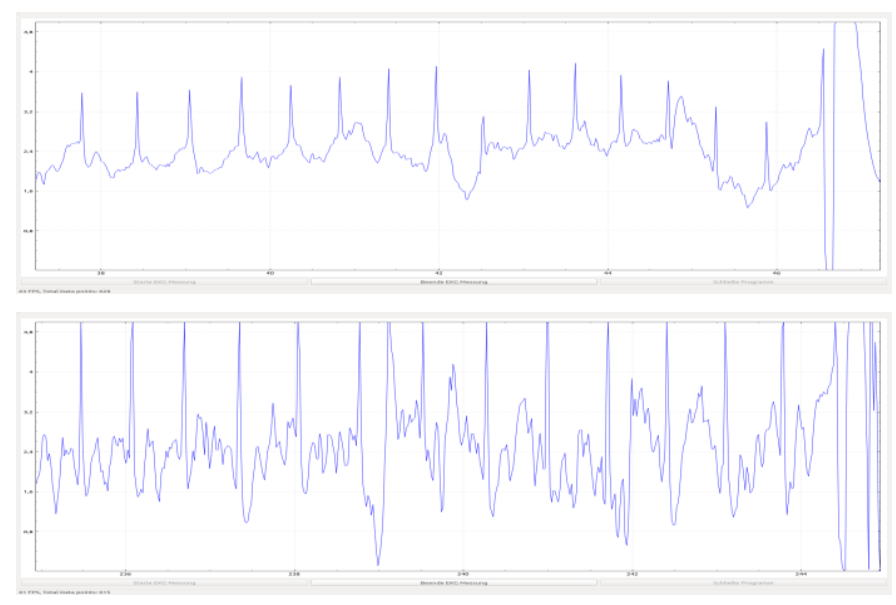

Fig. 10. Proposed ECG measurement signal through cloths on the chest according to Eindhoven I lead; top using PS25203B electrode, bottom using 25201B electrode.

\section{CONCLUSSIONS}

In this paper a novel, low cost capacitive ECG system has been designed, developed and compared with an earlier one [8]. The main differences in the hardware comprise the different microcontroller shields used. Due to the fact that the ECG shield from Olimex costs around 5 times less than the eHealth shield from Cooking Hacks, it comprises an optimum option for a pure capacitive ECG System. Furthermore, the two different electrodes from Plessey have been compared. Both, PS2503B and 25201B, can measure over direct skin contact (as a kind of dry electrode), as well as through cloths. PS2503B presented a better performance regarding noise, 
whereas PS2501B is able also to measure easier through several cloths layers. Digital filtering was found to slightly improve the acquired signal, however for the proposed system not appropriate enough, in order to filter dominant noise components (i.e. caused by power supply grid). The authors conclude, as it is also found in [28] that the shielding is the most promising solution in order to improve the signal performance regarding noise.

The XBee Pro Series 2B modules provide a secure opportunity to transfer the captured ECG signal to another computer or server in a real time manner. However, the sample frequency of the Arduino microcontroller platform is strongly decreasing via the utilized XBee communication modules. If the signal quality must be robust enough to not even loose a single $\mathrm{R}$ wave in the ECG signal, the XBee modules should not be directly attached to the Arduino board. Otherwise the sample frequency over USB and over XBee is dropping to approximately $25 \mathrm{~Hz}$. As the Arduino platform is not supporting multi-threading [25], the proposed solution of housing the data transfer routines on the BeagleBone Black platform, comprises the optimum solution, as the USB signal has $\sim 50 \mathrm{~Hz}$ and at the same time $\sim 25 \mathrm{HZ}$ sample frequency by XBee transmission.

As the ECG Shield, as well as the e-Health shield, are both originally designed for normal passive electrodes, the proposed system, can be used with standard ECG electrodes. The proposed hardware configuration, consisting of the Arduino/Wattuino Uno, attached to the Olimex ECG shield, which is further interfaced to the Plessy capacitive electrodes (PS25201B), it was found to comprise the most promising set up, in order to build a low cost capacitive ECG system, which is be able to measure through cloths.

\section{References}

[1] J. K Ninomiya., G. L'Italien, M. H. Criqui, J. L. Whyte, A. Gamst, and R. S. Chen, "Association of the metabolic syndrome with history of myocardial infarction and stroke in the Third National Health and Nutrition Examination Survey", Circulation, vol. 109, no. 1, pp. 42-46, 2004.

[2] D. Mozaffarian, E. J. Benjamin, A. S. Go, D. K. Arnett, M. J. Blaha, M. Cushman, S. Ferranit, J. P. Després, et al., "Executive Summary: Heart Disease and Stroke Statistics-2015 Update A Report From the American Heart Association", Circulation, vol. 131, no. 4, pp. 434-441, 2015.

[3] R. Kones, "Primary prevention of coronary heart disease: integration of new data, evolving views, revised goals, and role of rosuvastatin in management. A comprehensive survey", A comprehensive survey. Drug Des Devel Ther, vol. 5, pp. 325-80, 2011.

[4] D. J. Schofield, and J. R. Beard, "Baby boomer doctors and nurses: demographic change and transitions to retirement", Medical Journal of Australia, vol. 183, no. 2, pp. 80, 2005.

[5] M. J. McGrath, and C. N. Scanaill, "Wellness, fitness, and lifestyle sensing applications," in Sensor Technologies, Apress, pp. 217-248, 2013.

[6] J. Güttler, C. Georgoulas, T. Linner, and T. Bock, "Unabhängiges Wohnen durch modulare und mit Assistenzfunktionen individualisierbare Mikro-Roboterräume," Wohnen-Pflege-Teilhabe„Besser leben durch Technik“, 2014.

[7] T. Linner, W. Pan, C. Georgoulas, B. Georgescu, J. Güttler, and T. Bock, "Co-adaptation of robot systems, processes and in-house environments for professional care assistance in an ageing society," Procedia Engineering, vol. 85, pp. 328-338, 2014.

[8] J. Güttler, T. Linner, T. Bock, C. Georgoulas, "Development of a modular low cost contactless capacitive ECG including wireless data transfer," unpublished.

[9] S. Leonhardt, and A. Aleksandrowicz, "Non-contact ECG monitoring for automotive application," in Medical Devices and Biosensors, 2008, ISSS-MDBS 2008, 5th International Summer School and Symposium on IEEE, pp. 183-185, June 2008.

[10] M. Walter, B. Eilebrecht, T. Wartzek, and S. Leonhardt, "The smart car seat: personalized monitoring of vital signs in automotive applications," Personal and Ubiquitous Computing, vol. 15, no. 7, pp. 707-715, 2011.

[11] T. Wartzek, B. Eilebrecht, J. Lem, H. J. Lindner, S. Leonhardt, and M. Walter, "ECG on the road: robust and unobtrusive estimation of heart rate," Biomedical Engineering, IEEE Transactions on, vol. 58, no. 11, pp. 3112-3120, 2011.

[12] A. Arcelus, M. Sardar, and A. Mihailidis, "Design of a capacitive ECG sensor for unobtrusive heart rate measurements," in Instrumentation and Measurement Technology Conference (I2MTC), IEEE International, pp. 407-410, May 2013.

[13] Burr-Brown Products from Texas Instruments, OPA602 High-Speed Precision Difet OPERATIONAL AMPLIFIER; SBOS155A, August 1987, revised October 2002.

[14] Plessey, Epic... Monitoring Driver Health, http://www.plesseysemiconductors.com/epic-automotive-plesseysemiconductors.php, last access 08.02.2016.

[15] Plessey semiconductors, PS25203B EPIC Ultra High Impedance ECG Sensor Advance Information, Data Sheet 291499 issue 3.

[16] Plessey semiconductors, PS25201B EPIC Ultra High Impedance ECG Sensor Advance Information, Data Sheet 291497 issue 3.

[17] A. Aleksandrowicz, M. Walter, and S. Leonhardt, "Ein kabelfreies, kapazitiv gekoppeltes EKG-Messsystem/Wireless ECG measurement system with capacitive coupling," Biomedizinische Technik, vol. 52 no. 2, pp. 185-192, 2007.

[18] XBee/XBee-Pro ZB RF Modules, ZigBee RS Modules by Digi International, XBee ZB User Manual, 90000976_K, March 2012.

[19] Cooking hacks, e-Health Sensor Platform V1.0 for Arduino and Raspberry Pi [Biometric / Medical Applications], last access 08.02.2016, website: https://www.cookinghacks.com/documentation/tutorials/ehealth-v1-biometric-sensorplatform-arduino-raspberry-pi-medical

[20] Olimex, Shield-EKG-EMG, last access 08.02.2016, website: https://www.olimex.com/Products/Duino/Shields/SHIELD-EKG-EMG/

[21] G. Coley, "BeagleBone Black System Reference Manual-Revision C.1", 2014.

[22] QCustomPlot, website: http://www.qcustomplot.com/, last access 08.02.2016

[23] LOGIC supply, XBEE CAPE MANUAL - BeagleBone Black XBee Prototyping Cape, Revision 1.0, 2014.

[24] H. M. Piper, „Herzerregung“ in Physiologie des Menschen, pp. 517-626, Springer Berlin Heidelberg, 2011.

[25] H. Yamamoto, T. Fujii, P. T. T. Ha, and K. Yamazaki, "New development of remote control system for air vehicle using $3 \mathrm{G}$ cellular network," in Advanced Communication Technology (ICACT), 2014 16th International Conference on IEEE, pp. 456-461, February 2014.

[26] OLIMEX, Shield-EKG-EMG bio-feedback shield, User's Manual, Revision E, June 2014.

[27] W. H. Press, S. A. Teukolsky, W. T. Vetterling, and B. P Flannery, Numerical Recipes in C: The Art of Scientific Computing, $2^{\text {nd }}$ Edition. Cambridge University Press, 1992.

[28] T. Komensky, M. Jurcisin, K. Ruman, O. Kovac, D. Laqua, and P. Husar, "Ultra-wearable capacitive coupled and common electrode-free ECG monitoring system," in Engineering in Medicine and Biology Society (EMBC), 2012 Annual International Conference of the IEEE, pp. 1594-1597, August 2012. 